\title{
Efecto de diferentes niveles de interferencia en la fila de plantación en parámetros de crescimento en Eucalyptus grandis en Uruguay
}

\author{
Effect os different levels of interference in the planting \\ row in growth in Eucalyptus grandis in Uruguay
}

Juana Teresa Villalba ${ }^{1}$

\begin{abstract}
Resumo
El sector forestal en Uruguay ha tenido una expansión importante, con un incremento del área plantada de $158 \%$ en los últimos 15 años. Este aumento ha sido acompañado por un aumento en la productividad, generado por varias prácticas silvícolas, siendo una de ellas el control de malezas. Los efectos de la interferencia de malezas en los distintos parámetros de crecimiento dependen del sitio, de la silvicultura y de las condiciones climáticas. El objetivo del trabajo fue evaluar el efecto de diferentes niveles de interferencia de una especie competidora en la fila de plantación, sobre los parámetros de crecimiento de Eucalyptus grandis. El diseño experimental fue de bloques completos al azar con 3 repeticiones, los tratamientos consistieron en la siembra de diferentes densidades de moha (Setaria italica) en la fila de plantación, siendo ésta utilizada como especie competidora. Se analizó a través de modelos no lineares, el efecto de las densidades reales de moha (expresados en $\mathrm{n}^{\circ}$ de tallos $/ \mathrm{m}^{2}$ ) en la altura, diámetro de copa y diámetro a la altura de cuello a los 54, 78, 133 y 254 días post plantación (DPP). Hasta los 78 DPP no hubo efecto de la competencia excepto para diámetro de copa. En diámetro de copa y diámetro a la altura de cuello presentaron mayoritariamente un comportamiento exponencial negativo, a los 133 y a los 254 DPP. Mientras que la altura a los 133 DPP presentó un ajuste de tipo lineal. La comparación de tasas de decrecimiento para los distintos rangos de densidades de malezas, indicaron que el parámetro de mayor sensibilidad fue el diámetro de copa, con una disminución de $7.7 \mathrm{~cm}$ cada 10 tallos de moha.
\end{abstract}

Palabras clave: interferencia, malezas, Eucalyptus grandis

\begin{abstract}
The forestry sector in Uruguay has had a significant expansion, with an increase of the planted area of $158 \%$ in the last 15 years. This increase has been accompanied by an increase in productivity, generated by silvicultural practices, one of them being weed control. The effects of weed interference on the different growth parameters depend on the site and the climatic conditions. The objective of the work was to evaluate the effect of different levels of interference of a competing species in the plantation row, on the growth parameters of Eucalyptus grandis. The experimental design was randomized complete blocks with 3 replications, the treatments consisted in the planting of different densities of moha (Setaria itálica) in the row of plantation, being this used as a competing specie. The effect of the real moha densities (expressed in number of stems $/ \mathrm{m}^{2}$ ) on the height, tree top diameter and stem diameter at $54,78,133$ and 254 days post plantation, was analyzed through non-linear models. Up to 78 DPP there was no competition effect except for tree top diameter. In tree top diameter and stem diameter presented a negative exponential at 133 and 254 DPP. While the height at 133 DPP presented a linear type adjustment. The comparison of rates of decrease for the different ranges of weed densities, indicated that the parameter of greater sensitivity was the tree top diameter, with a decrease of $7.7 \mathrm{~cm}$ per 10 stems of Moha.
\end{abstract}

Keywords: interference, weeds, Eucalyptus grandis

\section{INTRODUCCIÓN}

El sector forestal en Uruguay ha tenido una expansión muy grande, el crecimiento de la superficie plantada en los últimos 15 años fue de $158 \%$, con la presencia de empresas extranjeras que han realizado grandes inversiones a nivel de plantaciones e industrias. El principal género plantado es Eucalyptus, alcanzando el 73\% del área forestada, siendo la pasta de celulosa el principal producto de exportación del sector (MGAP, 2016).

1Professora Doutora. Universidad de la Republica / Faculdade de Agronomia. Estación Experimental Dr. Mario Cassinoni. Ruta 3 Km 363 - Paysandú, Uruguay. E-mail: villalba@fagro.edu.uy.

Sci. For., Piracicaba, v. 46, n. 119, p. 473-482, set. 2018 DOI: dx.doi.org/10.18671/scifor.v46n119.14 
La expansión fue acompañada por el aumento de producción consecuencia de la mejora genética y de las prácticas silvícolas y es la interferencia de malezas, uno de los principales problemas que afronta la silvicultura.

El control de malezas en las plantaciones de eucaliptos en Uruguay contemplan las aplicaciones de herbicidas totales previo a la plantación y luego se realiza el control de las malezas en la fila y más tarde el control en la entre fila. Para el control de malezas en la fila se usan herbicidas selectivos, premergentes y/o postemergentes y glifosato en aplicaciones dirigidas, mientras que el control de malezas de la entre fila es con glifosato. En ambos casos el número de intervenciones es variable según el enmalezamiento y según cuando ocurra el cierre de copa del monte (VILLALBA, 2011).

La presencia de malezas en ecosistemas forestales, ha sido un problema importante en la instalación y mantenimiento de las plantaciones de eucaliptos, esto se ha comprobado mediante estudios que indican que las malezas coexistiendo con el monte pueden afectar negativamente el crecimiento y productividad de los mismos (COSTA et al., 2004; GARAU et al., 2009; TOLEDO et al., 2003). Incluso con disminuciones en la sobrevivencia (APARICIO et al., 2005; HUERTA et al., 2012; TOLEDO et al., 2000). Mientras que en plantaciones de segundo corte no se han registrado efectos (SOUZA et al., 2010).

La interferencia ejercida por las malezas en plantaciones de eucalipto es de mayor importancia en las primeras etapas de crecimiento (COSTA et al., 2004). Los efectos adversos son directamente proporcionales al periodo de tiempo en que estas coexisten (ADAMS et al., 2003; COSTA et al. 2004). En plantaciones de eucalipto en diferentes regiones de Brasil, los periodos críticos de competencia han variado, desde 20 días (COSTA et al., 2004), 78 días (TOLEDO et al., 2003) y 112 días (DINARDO et al., 1998).

La competencia es capaz de ejercer una notable influencia sobre las diferencias existentes entre individuos, siendo que pequeñas diferencias iniciales pueden dar lugar a variaciones drásticamente mayores con el tiempo (PARUELO; BATISTA, 2006). Así es que la interferencia en eucalipto puede resultar en una pérdida del rendimiento final de madera, asociado a esto un aumento en el tiempo de rotación (LITTLE; SCHUMANN, 1996), así como en un marcado efecto en la homogeneidad del monte (LAROCCA et al., 2004).

Los parámetros de crecimiento afectados por la interferencia son, altura, diámetro de los árboles, área foliar, volumen individual, crecimiento radicular, aunque algunos autores destacan que el parámetro más afectado es el diámetro (DINARDO et al., 1998; GARAU et al., 2009; SCHALLER et al., 2003 STONE; BIRK, 2001; TOLEDO et al., 2003). También se ha observado una disminución en el potencial hídrico foliar y la conductancia estomática con el aumento de la densidad de malezas (GARAU et al., 2008).

Como mecanismo de respuesta a la presencia de malezas, a los cambios de la calidad de luz que reciben, relación rojo-rojo lejano, las plantas realizan ajustes en su morfología (RAJCAN et al., 2004). En el caso de eucalipto tienden a perder rápidamente ramas y hojas de la porción basal; presentando una menor cantidad total de las mismas concentradas en el ápice de la planta. Lo anterior es provocado por una competencia por luz, que promueve una elongación pronunciada de la planta (TOLEDO et al., 2003).

La competencia por un recurso afecta a menudo la capacidad de explotar otros recursos. Por eso la reducción de la intercepción de luz por sombreado de la competidora afectará su tasa de crecimiento radical, y consecuentemente, su capacidad de usar recursos del suelo se verá afectada (PARUELO; BATISTA, 2006; SCHALLER et al, 2003), estos efectos son más marcados en años con deficiencias hídricas (SCHALLER et al., 2003).

Una forma frecuente de estudiar los efectos de la competencia es comparar situaciones en las que se varía artificialmente la densidad. A medida que la densidad aumenta la abundancia relativa de los recursos disminuye, y el grado de competencia aumenta (PARUELO; BATISTA 2006).

La especie usada como competidora fue Setaria itálica, es una especie estival, de gran competitividad, C4, usada en el país como especie forrajera (TERRA et al., 2000).

El objetivo de este trabajo fue evaluar el efecto de diferentes niveles de interferencia de una especie competidora en la fila de plantación, sobre los parámetros de crecimiento de Eucalyptus grandis en las condiciones de crecimiento de Uruguay. 


\section{MATERIALES Y MÉTODOS}

El experimento se instaló en un predio propiedad de Forestal Oriental- S.A.- UPM, Establecimiento San Luis, situado al Este de la ciudad de Paysandú, Uruguay.

La historia del establecimiento donde se realizó el experimento correspondía a rotación forestal, con suelos del grupo CONEAT 9.3 (MGAP, 2017). Estos suelos forman parte de la Asociación de Suelos Algorta. Los suelos predominantes corresponden a Planosoles Dístricos Ócricos, a veces Melánicos y Argisoles Dístricos Ócricos Abrúpticos, a veces Típicos. Luego de la cosecha y 20 días previo a la instalación y plantación del experimento se realizó la aplicación de glifosato en área total con una dosis de 1800 g.ea/ha. La plantación se realizó con mudas de Eucalyptus grandis propias de la empresa y la fertilización fue con $90 \mathrm{~g}$ por planta de Fosfato Diamónico. El marco de plantación correspondió a una distancia entre plantas de 2.75 metros y entre filas de $3 \mathrm{~m}$.

El diseño experimental fue de bloques completos al azar con 3 repeticiones, el tamaño de la unidad experimental fue de 2 filas de 10 árboles cada una. Los tratamientos consistieron en niveles de competencia planteados a través de la siembra en la fila de plantación de las densidades, 0, 20, 40, 60 y 80 semillas de moha (Setaria itálica), usada como especie competidora.

Se analizó el efecto de las densidades reales sobre el crecimiento de los árboles a través de análisis de regresión no lineal y considerando cada parcela en forma independiente con la densidad real de la especie competidora. Debido al profuso macollaje de la especie competidora (Setaria itálica), las densidades reales no fueron las planteadas inicialmente, por ello se realizó el conteo de tallos en 3 muestreos de $30 \times 30 \mathrm{~cm}$ por cada fila y estas densidades reales se expresaron en número de tallos. $\mathrm{m}^{-2}$.

Los parámetros de altura, diámetro a la altura del cuello y diámetro de copa de cada árbol fueron medidos al momento de la plantación, a los 54, 78, 133 y 254 días post- plantación (DPP).

Las variables diámetro a la altura del cuello y diámetro de copa ajustaron a un modelo exponencial negativo a los 133 y 254 DPP, al igual que la altura en la evaluación de los 254 DPP. Las variables diámetro de copa a los 78 DPP y altura a los 133 DPP se ajustaron a un modelo de regresión lineal simple.

Los datos climáticos del periodo experimental se presentan en el Cuadro 1, juntamente con el promedio histórico para la región (datos de Dirección Nacional de Meteorología, Uruguay).

Cuadro 1. Datos meteorológicos del sitio del experimento y del promedio histórico de la región para el periodo del experimento.

Table 1. Meteorological data of the experiment site and the historical average of the region for the period of the experiment.

\begin{tabular}{lcccc}
\hline Mes & $\begin{array}{c}\text { Temperatura } \\
\text { mínima ( }\end{array}$ & $\begin{array}{c}\text { Temperatura } \\
\text { máxima }\left({ }^{\circ} \mathbf{C}\right)\end{array}$ & $\begin{array}{c}\text { Precipitaciones } \\
\text { registradas (mm) }\end{array}$ & $\begin{array}{c}\text { Precipitaciones } \\
\text { promedio histórico }(\mathbf{m m})\end{array}$ \\
\hline Setiembre & 7,3 & 20,6 & 98,0 & 91 \\
Octubre & 9,0 & 21,5 & 372,0 & 122 \\
Noviembre & 12,0 & 27,6 & 20,0 & 118 \\
Diciembre & 13,6 & 32,2 & 262,0 & 115 \\
Enero & 16,4 & 32,4 & 73,0 & 100 \\
Febrero & 14,6 & 29,7 & 130,0 & 131 \\
Marzo & 11,6 & 25,9 & 138,0 & 147 \\
Abril & 10,2 & 26,2 & 65,0 & 103 \\
Mayo & 7,9 & 21,1 & 198,0 & 77 \\
\hline
\end{tabular}

\section{RESULTADOS Y DISCUSIÓN}

Los resultados del efecto de la interferencia se presentan para cada parámetro y se comparan las respuestas en las distintas fechas de evaluación.

El dato general y más relevante es que las diferentes densidades de la especie competidora no afectaron la sobrevivencia de los árboles. Esto puede explicarse principalmente por el régimen de precipitaciones, que en el acumulado del periodo experimental fue 35\% superior al promedio histórico para la región en igual periodo. Concordantemente, algunos autores mencionan que el factor abiótico que afecta principalmente a la supervivencia y crecimiento de las plantaciones forestales es la disponibilidad hídrica y uno de los efectos principales de las malezas es que pueden causar estrés 
hídrico (GARAU et al., 2008). Las malezas son principales competidores por el recurso hídrico y son los árboles jóvenes los más susceptibles al estrés hídrico en los primeros meses después de la plantación (GARAU et al., 2009, 2008).

En la primera fecha de evaluación 54 DPP, para todos los parámetros evaluados, no fue posible el ajuste de ningún modelo. La inexistencia de respuesta a la competencia de malezas hasta esa fecha, se puede explicar por el tiempo de convivencia. Concordantemente, Garau et al. (2009) y Toledo et al. (2003) indicaron que los primeros efectos negativos más evidentes de competencia en el crecimiento de los eucaliptos se registraron a partir del segundo mes de convivencia.

Por otra parte, considerando las precipitaciones acumuladas desde implantado el experimento hasta la fecha evaluada, se registró un valor acumulado de $490 \mathrm{~mm}$, esto es un $48 \%$ superior al promedio histórico. Este indicador nos permite inferir que en esta instancia el agua no fue limitante para el crecimiento de los eucaliptos, García et al. (2015) mencionan que el Eucalyptus grandis tiene un requerimiento mínimo de precipitación anual de $1020 \mathrm{~mm}$, es decir que en este período ya se había alcanzado la mitad del requerimiento hídrico, siendo este capaz de cubrir las necesidades de los árboles tanto como de las malezas.

A los $78 \mathrm{DPP}$, solamente fue posible el ajuste de diámetro de copa a un modelo de regresión lineal simple $(\mathrm{P}<0.0001)$. La ecuación del diámetro de copa para los números de tallos (D) de la especie competidora fue $\mathrm{Y}=54.72-0.0542 \times \mathrm{D}$ (densidad de tallos). Esta estimación permite afirmar que en este período cada 10 plantas en competencia con el árbol se generó una pérdida de 0.5 cm en el diámetro de copa.

Para las fechas de 133 y 254 DPP la respuesta por el efecto de la competencia permitió el ajuste al modelo exponencial negativo. Se analizó la tasa de decrecimiento de la variable en función de la densidad. Las tasas de decrecimiento (parámetro $\mathrm{C}$ ) de ambos periodos fueron significativas y fueron similares, ya que los intervalos de confianza se superpusieron (Cuadro 2).

Cuadro 2. Medias de diámetro de copa, parámetro C (tasa de decrecimiento para las densidades de maleza), p-valor de $\mathrm{C}$ y límites de confianza.

Table 2. Average tree top diameter, $C$ parameter, $p$-value of $C$ and confidence limits.

\begin{tabular}{lccccc}
\hline DPP & Media & p-valor & C & \multicolumn{2}{c}{ Límites de confianza } \\
\cline { 4 - 6 } & 84.8 & 0.005 & -0.0075 & Límite inferior & Límite superior \\
\hline 133 & 138.6 & 0.037 & -0.0104 & -0.01260 & -0.00243 \\
254 & & & -0.02020 & -0.00066 \\
\hline
\end{tabular}

Las curvas de comportamiento construidas a partir de los datos del modelo se presentan en la Figura 1. Estos resultados eran esperados, ya que se ha alcanzado un período de convivencia importante entre malezas y eucalipto como para que ocurra la expresión de estos efectos negativos. Pudiendo en este caso, el agua afectar de cierta manera el crecimiento de los árboles al encontrarse menos disponible que en el período anterior. Según Garau et al. (2009), esta es una variable muy sensible a la competencia de malezas, estos autores obtuvieron una reducción del $95 \%$ en el área foliar, variable asociada al diámetro de copa, aun con 25\% de cobertura de malezas.

En el diámetro a la altura de cuello fue posible el ajuste a modelo exponencial negativo para las fechas 133 y 254 DPP (Cuadro 3, Figura 2).

Cuadro 3. Medias de diámetro a la altura de cuello, parámetro C, p-valor de C y límites de confianza.

Table 3. Average of stem diameter, parameter $C, p$-value of $C$ and confidence limits.

\begin{tabular}{lccccc}
\hline DPP & Media & p-valor & C & \multicolumn{2}{c}{ Límites de confianza } \\
\cline { 5 - 6 } & 16.24 & 0.039 & -0.0056 & Límite inferior & Límite superior \\
\hline 133 & 34.2 & 0.039 & -0.0086 & -0.01080 & -0.00027 \\
254 & & & -0.01670 & -0.00048 \\
\hline
\end{tabular}

Al igual que en diámetro de copa las tasas de decrecimiento fueron significativas y no fueron diferentes. 


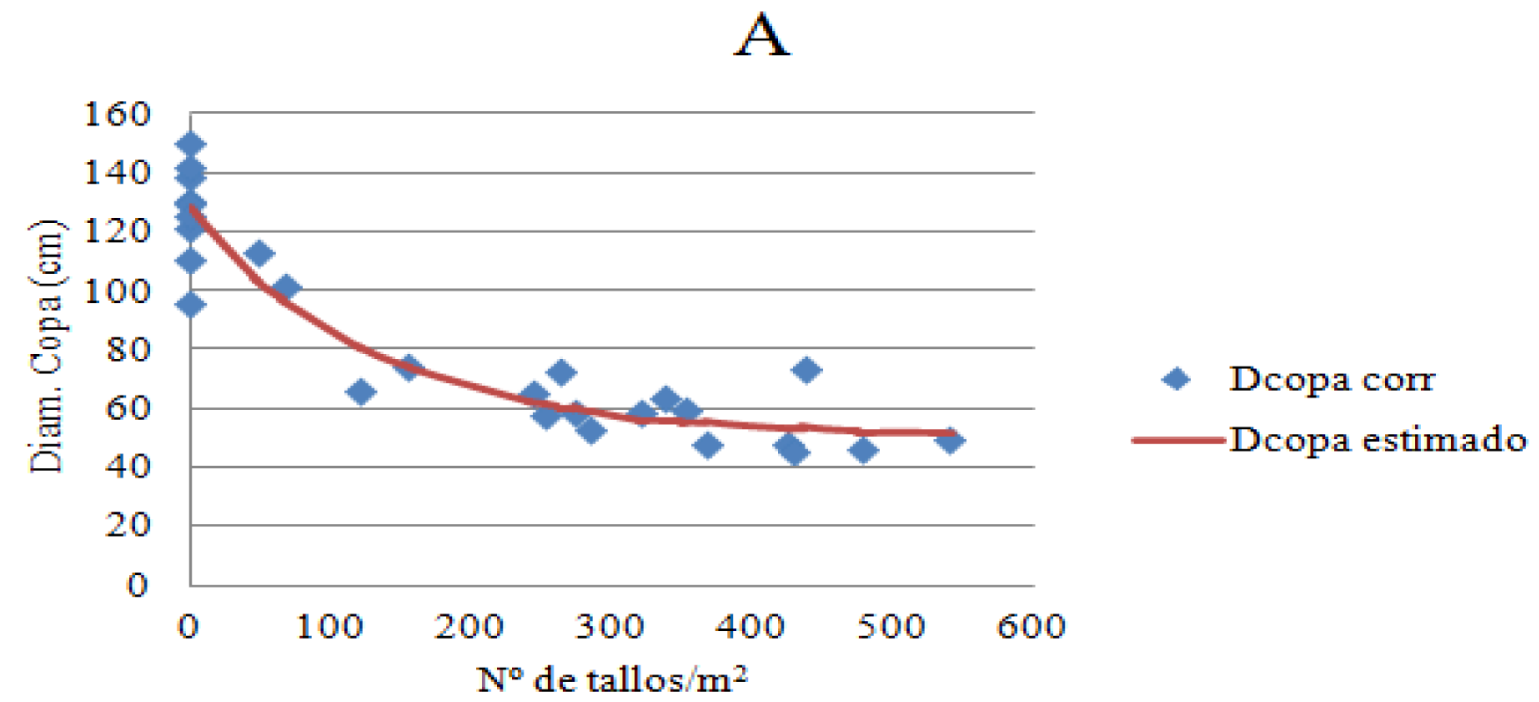

B

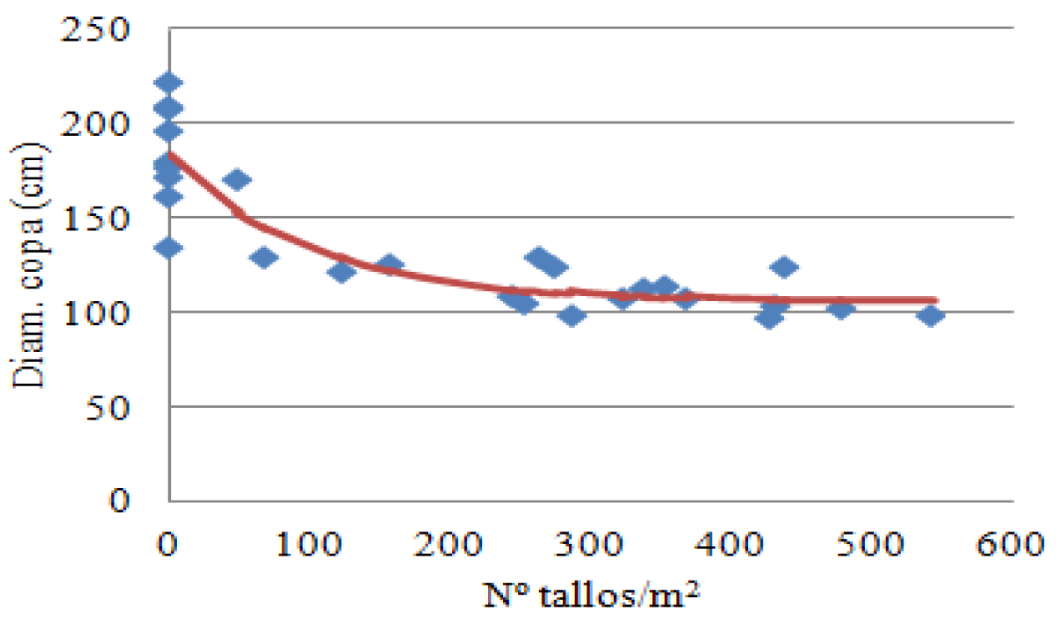

- Dcopa corr

Dcopa estimado

Figura 1. Curvas ajustadas del diámetro copa en función de la densidad de tallos $/ \mathrm{m}^{2}$ de moha en las fechas A) 133 DPP; B) 254 DPP.

Figure 1. Adjusted curves of the tree top diameter as a function of the stems density/ $\mathrm{m}^{2}$ of moha in the dates $A$ ) 133 DPP; B) 254 DPP.

El diámetro es una variable de gran influencia en el volumen a turno final, lo que traería como consecuencia, una reducción en el volumen total y comercial, ya que la industria de celulosa exige un diámetro mínimo para su procesamiento de $5 \mathrm{~cm}$, y al existir dentro de la plantación, árboles con alturas similares, ese diámetro va a presentarse a diferentes alturas, por lo tanto además de disminuir el volumen total, el aprovechamiento de ese volumen a nivel comercial será menor, aumentando los desperdicios a nivel de campo, y generando pérdidas económicas. 
Villalba et al. - Efecto de diferentes niveles de interferencia en la fila de

plantación en parámetros de crescimento en Eucalyptus grandis en Uruguay

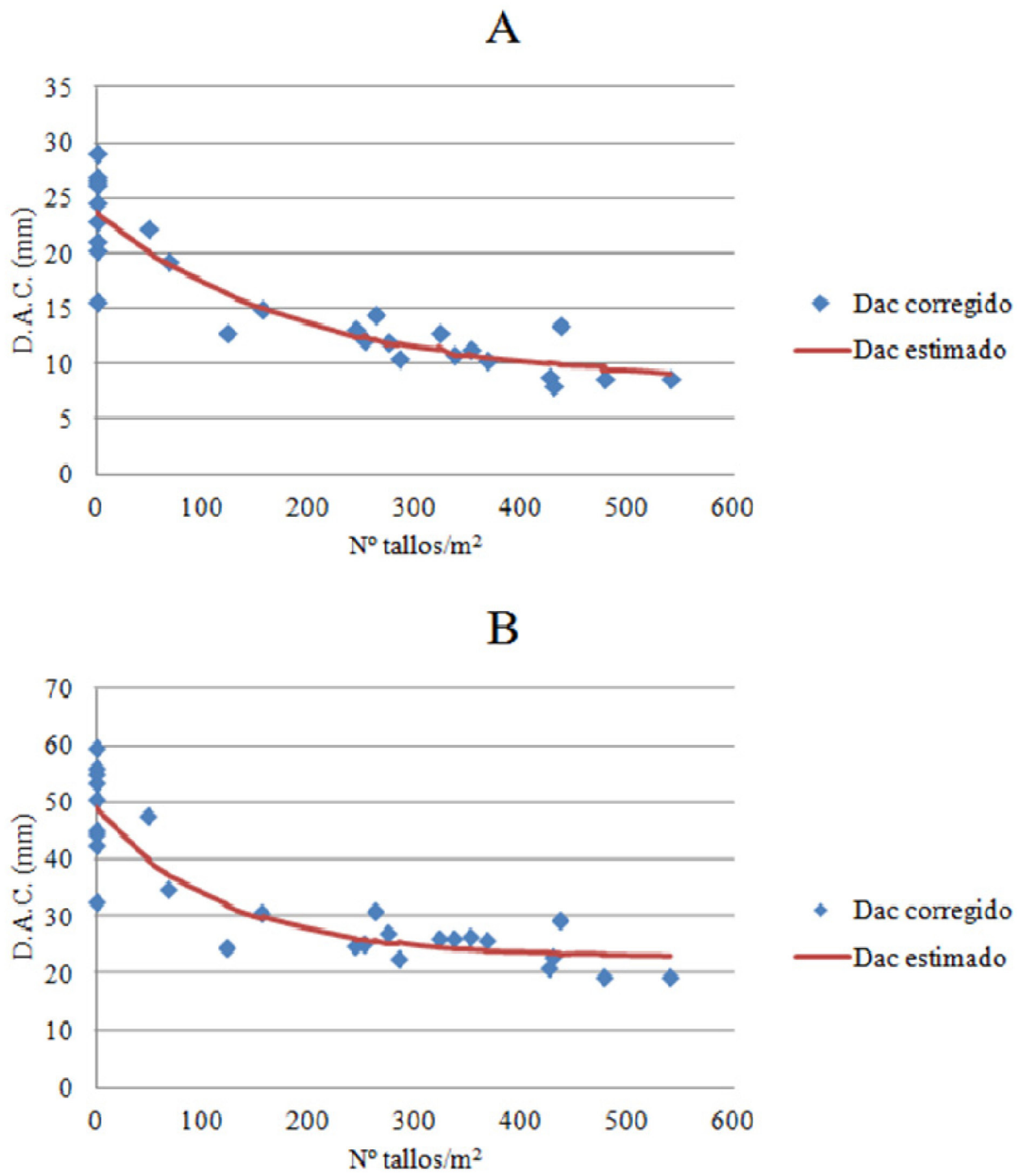

Figura 2. Curvas ajustadas de diámetro de altura de cuello en función de la densidad de tallos/ $\mathrm{m}^{2}$ de moha en las fechas A) 133 DPP; B) 254 DPP.

Figure 2. Curves adjusted stem diameter as a function of stem density/m² of moha in the dates A) 133 DPP; B) 254 DPP.

La altura permitió un ajuste a un modelo de regresión lineal simple ( $\mathrm{Y}=161.044-0.1421$ x D) a los 133 DPP y a modelo exponencial negativo a los 254 DPP (Cuadro 4 y Figura 4).

Cuadro 4. Medias de altura, parámetro $C$, $p$-valor de $C$ y límites de confianza.

Table 4. Average height, parameter $C, p$-value of $C$ and confidence limits.

\begin{tabular}{lccccc}
\hline \multirow{2}{*}{ DPP } & Media & p-valor & C & \multicolumn{2}{c}{ Límites de confianza } \\
\cline { 4 - 6 } & & & Límite inferior & Límite superior \\
\hline 254 & 243.6 & 0.098 & -0.0053 & -0.0160 & 0.00107 \\
\hline
\end{tabular}


A diferencia de las variables diámetro a la altura del cuello y diámetro de copa, el aumento de la densidad de malezas no provocó un decrecimiento de la altura significativo, solo hubo una tendencia. Esto concuerda con lo mencionado por varios autores, respecto a que la altura no es el parámetro más afectado por la interferencia de malezas. La competencia por luz promueve una elongación pronunciada de la planta que puede explicar la respuesta diferencial de esta variable. A campo, esto puede ocasionar una mayor probabilidad de vuelco ante la ocurrencia de vientos fuertes, ya que árboles altos con diámetros finos es esperable que presenten problemas de anclaje.
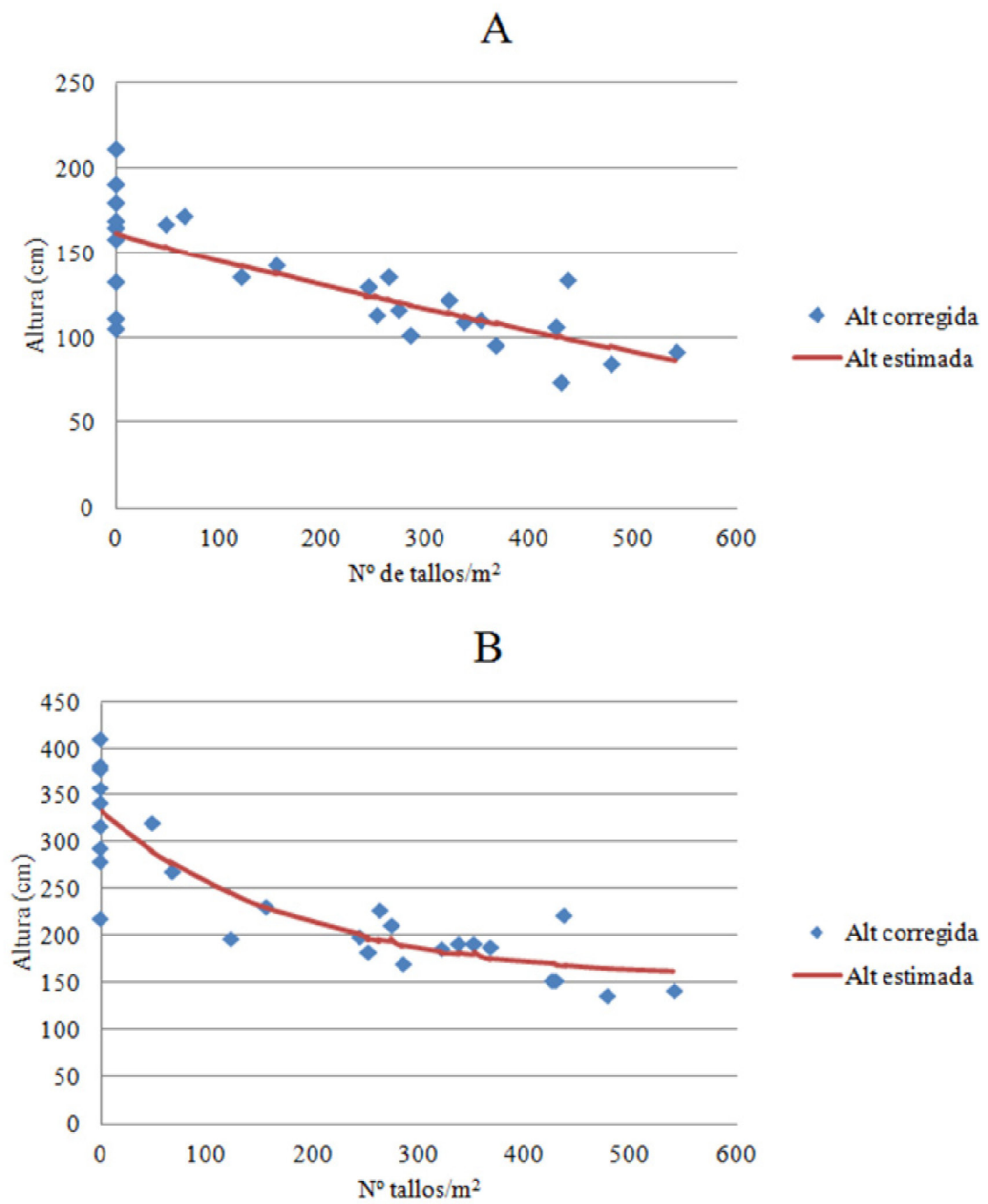

Figura 3. Curvas ajustadas de altura en función de la densidad de tallos $/ \mathrm{m}^{2}$ de moha en las fechas A) 133 DPP; B) 254 DPP.

Figure 3. Height-adjusted curves as a function of stem density $/ \mathrm{m}^{2}$ of moha in the dates A) 133 DPP; B) 254 DPP.

Usando los parámetros de los diferentes modelos ajustados para las variables en estudio en las distintas fechas, se estimaron las pérdidas que generan diferentes densidades de malezas y se aproximaron para 5, 50, 100 y 200 plantas por $\mathrm{m}^{2}$ (Cuadro 5). 
Villalba et al. - Efecto de diferentes niveles de interferencia en la fila de

plantación en parámetros de crescimento en Eucalyptus grandis en Uruguay

Cuadro 5. Estimación de tasa de pérdida $(\mathrm{cm}) / 10$ tallos de moha, en las distintas variables para diferentes rangos de densidades de malezas.

Table 5. Estimation of loss rate $(\mathrm{cm}) / 10$ stems of moha, in the different variables for different ranges of weed densities.

\begin{tabular}{lcc|ccc}
\hline \multirow{2}{*}{$\mathbf{N}^{\circ}$ tallos $/ \mathbf{m}^{2}$} & \multicolumn{2}{c|}{ 133 DPP } & \multicolumn{2}{c}{ 254 DPP } \\
\cline { 2 - 6 } & $\begin{array}{c}\text { Diámetro } \\
\text { de copa }\end{array}$ & $\begin{array}{c}\text { Diámetro a la } \\
\text { altura de cuello }\end{array}$ & $\begin{array}{c}\text { Diámetro } \\
\text { de copa }\end{array}$ & $\begin{array}{c}\text { Diámetro a la } \\
\text { altura de cuello }\end{array}$ & Altura \\
\hline 5 & 5.5 & 0.8 & 7.7 & 2.1 & 9.3 \\
50 & 4.0 & 0.6 & 4.7 & 1.4 & 7.4 \\
100 & 2.8 & 0.5 & 3.0 & 0.9 & 5.5 \\
200 & 1.3 & 0.3 & 1.0 & 0.4 & 3.2 \\
\hline
\end{tabular}

Estas estimaciones nos permiten identificar las tasas de decrecimiento para distintos rangos de malezas en competencia, y ver el impacto que tienen las bajas densidades comparadas con las más altas. A los 133 y 254 DPP se observó una tendencia muy similar, en las mayores densidades, de 100 y 200 plantas $/ \mathrm{m}^{2}$, cada unidad de maleza generó menores pérdidas (Cuadro 5).

Cabe destacar que este análisis fue sobre un ensayo que se realizó a 8 meses desde plantación, aún no se había alcanzado el cierre copa, momento a partir del cual los árboles comienzan el sombreado disminuyendo el efecto de las malezas, por lo tanto, es de esperar que las malezas siguieran interfiriendo en el desarrollo de la plantación, período que no fue analizado en este trabajo.

Para determinar la variable más sensible a la interferencia de malezas en aquellas evaluaciones que ajustaron al modelo exponencial negativo se compararon las tasas de decrecimiento, realizando la relación entre la estimación de la tasa (parámetro C) y su error estándar. Las relaciones indicaron que a los 133 y 254 DPP la variable más sensible fue el diámetro de copa comparada al diámetro a la altura de cuello (Cuadro 6).

Cuadro 6. Relación tasa de decrecimiento, C y su error estándar.

Table 6. Ratio rate of decrease, $\mathrm{C}$ and its standard error.

\begin{tabular}{lccc}
\hline DPP & altura & Diámetro de copa & Diámetro a la altura de cuello \\
\hline 133 & -- & 3.061 & 2.185 \\
254 & 1.725 & 2.213 & 2.194 \\
\hline
\end{tabular}

\section{CONCLUSIONES}

La interferencia de hasta 541 tallos $/ \mathrm{m}^{2}$ de la especie competidora moha (Setaria itálica), no generó efectos en la supervivencia de Eucalyptus grandis, hasta los 8 meses post- plantación y con un acumulado de precipitaciones del 35\% superior al histórico para ese período.

Hasta los 78 DPP el efecto de la competencia solo se manifestó en la disminución del diámetro de copa.

La disminución en el crecimiento de E. grandis presentó una tendencia a mayor pérdida de crecimiento cuanto mayor el periodo de convivencia con la maleza. Las pérdidas fueron superiores en las densidades más bajas, de 5 plantas $/ \mathrm{m}^{2}$, siendo la respuesta mayoritariamente de tipo exponencial negativo.

La comparación de los valores de las tasas de decrecimiento para los distintos rangos de densidades de malezas, indicó que el parámetro de mayor sensibilidad fue el diámetro de copa, seguido del diámetro de cuello y la altura.

\section{REFERENCIAS BIBLIOGRÁFICAS}

ADAMS, P. R.; BEADLE, C. L.; MENDHAM, N. J.; SMETHURST, P. J. The impact of timing and duration of grass control on growth of a young Eucalyptus globulus Labill. New Forests, Amsterdam, n. 26, p.147-165, 2003.

APARICIO, J. L.; LAROCCA, F.; DALLA TEA, F. Silvicultura de establecimiento de Eucalyptus grandis. IDIA XXI, Revista de Información y Desarrollo Agropecuario, v.5, n. 8, p.66-69, 2005. 
COSTA, A. G. F; ALVES, P. L. C. A.; PAVANI, M. C. M. D. Periodos de interferencia de trapoeraba (Commelina benghalensis Hort.) no crescimento inicial de eucalipto (Eucalyptus grandis W, Hill ex Maiden). Revista Árvore, Viçosa, n. 28, n. 4, p. 471-478, 2004.

DINARDO, W.; TOLEDO, R.; ALVES, P.; GALLI, A. Interferência da palhada de capim-braquiária, sobre o crescimento inicial de eucalipto. Planta Daninha, Viçosa, v.16, n 1, p. 13 -23, 1998.

GARAU, A. M.; GHERSA, C. M.; LEMCOFF, J. H.; BARAÑAO, J. J. Weed in Eucalyptus globulus subsp. Maidenii (F. Muell) establishment: effects of competition on sapling growth and survivorship. New Forests, Amsterdam, n. 37, p. 251-264, 2009.

GARAU, A. M.; LEMCOFF, J. H.; GHERSA, C. M.; BEADLE, C. L. Water stress tolerance in Eucalyptus globulus Labill. subsp. maidenii (F. Muell.) saplings induced by water restrictions imposed by weeds. Forest Ecology and Management, Amsterdam, n. 255, p. 2811-2819, 2008.

GARCÍA, E, R, SOTOMAYOR A. SILVA .S.VALDEBENITO G. Establecimiento de Plantaciones Forestales, 2015, 32 p. Disponível em: < http://icf.gob.hn/wp-content/uploads/2015/12/PREFO-Manual-del-Eucalipto. pdf >. Acesso em: 9 Mai 2017.

HUERTA, N.; MENENDEZ, J; MOTTA, S. Estudio de herbicidas residuales usados en pre plantación de Eucalyptus. 2008. 55 p. Tesis (Monografía de grado) - Facultad de Agronomía, Uruguay, 2008.

LAROCCA, F.; DALLA TEA, F; APARICIO, J. L. Técnicas de implantación y manejo de Eucalyptus grandis para pequeños y medianos forestas en Entre Ríos y Corrientes. 2004. Disponível em: < http://64.76.123.202/ new/0-0/forestacion/ archivos/ biblioteca/228\%20Larocc.pdf >. Acesso em: 06 Febr. 2016.

LITTLE, K. M.; SCHUMANN, A. W. A new systematic trial design for the optimization of interspecific weed control. In: Australian Weeds Conference Proceedings. 11., 1996. Proceedings... 1996. Disponível em: < http://caws.org.au/awc/1996/awc199614401.pdf >. Acesso em: 15 Mai 2017.

MGAP. Anuario estadístico agropecuario. Montevideo: Estadísticas Agropecuarias DIEA, 2016. 198 p.

MGAP. Descripción de los grupos de suelos CO.N.E.A.T. Montevideo: Estadísticas Agropecuarias DIEA 2017. Disponível em:< www.mgap.gub.uy/...de.../grupos-de-suelos-e-indices-de-productividad-coneat $>$ Acesso em: 15 Abr. 2017.

PARUELO, J. M.; BATISTA, W. El flujo de energía en los ecosistemas. In: Fundamentos de ecología. Buenos Aires: Universidad de Buenos Aires. Facultad de Agronomía, 2006. p. 97 - 115

RAJCAN, I.; CHANDLER, K. J.; SWANTON, C. J. Red-far-red-ratio of reflected light: a hypothesis of why earlyseason weed control is important in corn. Weed Science, Champaign, n. 52, n. 5, p. 774-778, 2004.

SCHALLER, M.; SCHROTH, G.; BEER, J.; JIMENEZ, F. Root interaction between young Eucalyptus deglupta trees and competitive grass species in contour strip. Forest Ecology and Management, Amsterdam, v. 179, p. 429$440,2003$.

SOUZA, M. C.; COSTA, P. L.; PEREIRA, T. Interferência da comunidade infestante sobre plantas de Eucalyptus grandis de segundo corte. Scientia Forestalis, Piracicaba, v. 38, n. 85, p. 63-71, 2010.

STONE, C.; BRIK, E. Benefits of weed control and fertiliser application to young Eucalyptus dunnii stressed from waterlogging and insect demage. Australian Forestry, Melbourne, v. 64, n. 3, p. 151-158, 2001.

TERRA, J. E.; SCAGLIA, G.; GARCÍA PRÉCHAC, F. MOHA: Características del cultivo y comportamento em rotaciones forrajeras com siembra directa. Serie Técnica INIA, n. 111, Inia, 2000. 62 p.

Sci. For., Piracicaba, v. 46, n. 119, p. 473-482, set. 2018 DOI: dx.doi.org/10.18671/scifor.v46n119.14 
Villalba et al. - Efecto de diferentes niveles de interferencia en la fila de

plantación en parámetros de crescimento en Eucalyptus grandis en Uruguay

TOlEDO, R. E. B.; VICTORIA, R.; BEZUTTE, A. J.; PITEllI, R. A.; AGUIAR, P. L.; VALlE, C.; ALVARENGA, S. F. Periodos de controle de Brachiaria sp. e seus reflexos na productividade de Eucalyptus grandis. Scientia Forestalis, Piracicaba, n. 63, p. 221-232, 2003.

TOlEDO, R. E. B.; VICTÓRIA, F. R.; PITElli, R. A.; ALVES, P. L. C. A.; LOPES, M. A. F. Efeito de períodos de controle de plantas daninhas sobre o desenvolvimento inicial de plantas de eucalipto. Planta Daninha. Viçosa, v. 18, n. 3, p. 395-404, 2000.

VILLALBA, J. Control de malezas en Eucalyptus spp. Serie Actividades de Difusión, INIA, Uruguay, v. 629, p. $1-8,2011$.

Recebido em 04/08/2017

Aceito em 12/03/2018 